\title{
HLA antigens in Behçet's disease: a reappraisal by a comparative study of Turkish and British patients
}

\author{
H. YAZICI, ${ }^{1}$ M. ANNE CHAMBERLAIN, ${ }^{2}$ IEKE SCHREUDER, ${ }^{3}$ \\ J. D'AMARO, ${ }^{4}$ AND M. MUFTUOGLU ${ }^{1}$
}

From the ${ }^{1}$ Department of Medicine, Carrahpasa Medical Faculty, University of Istanbul; the ${ }^{2}$ Department of Rheumatology, General Infirmary, Leeds, England; the ${ }^{3}$ Department of Immunohaematology, University Medical Centre, Leiden, Netherlands; and the ${ }^{4}$ Dutch Organisation for the Advancement of Pure Research $(Z W O)$

SUMMARY We have compared the distribution of histocompatibility antigens of 22 Turkish and 14 British patients with Behçet's disease with those of their respective controls. There was a strong association of B5, specifically its Bw51 split, with the disease. A modest increase in the incidence of HLA B27 was noted in British patients, but numbers were too small for analysis. The incidence of DRw2 and DRw4 antigens among Turkish and British patients did not differ from that of their respective controls.

Regional differences have been reported for the associations between the HLA antigens and Behçet's disease. Ohno et al. ${ }^{1}$ from Japan initially reported a strong association with HLA B5. Confirmatory reports appeared from Turkey, ${ }^{2}{ }^{3}$ Israel, ${ }^{4}$ southern France, ${ }^{5}$ Japan, ${ }^{6}$ and Northern Africa. ${ }^{7}$ Two reports from Britain ${ }^{8}{ }^{9}$ and 1 from the United States ${ }^{10}$ failed to find an association with any of the alleles tested. Another paper from Britain ${ }^{11}$ reported an association with HLA B5 in male patients only and a weak association in the entire group with HLA B27. None of those studies contained data on the DR locus antigens.

The purpose of the studies in this report was to re-examine the frequencies of the HLA antigens in patients with Behçet's disease and their controls in 2 Caucasoid populations, especially with regard to the newly defined splits of HLA $B 5^{12}$ and the HLA DR locus antigens which have not been studied previously.

\section{Materials and methods}

The probands came from Istanbul, Turkey, and from Leeds, England. Most of them had been tissue typed previously in the local laboratories.

Accepted for publication 31 July 1979

Correspondence to Dr Anne M. Chamberlain, School of Medicine, 36 Clarendon Road, Leeds LS2 9PJ.
They were called back for retyping on the basis of availability irrespective of the results of their previous typings.

The diagnostic criteria of Mason and Barnes ${ }^{13}$ had originally been used for the patients in Leeds and those of O'Duffy ${ }^{14}$ for those in Istanbul. For uniformity the entire group was re-evaluated according to O'Duffy's criteria. Four patients from the British group were excluded. There were 22 Turkish (19 males, 3 females) and 14 British (6 males, 8 females) patients that fulfilled the criteria.

Unrelated healthy laboratory technicians, medical students, house staff, and faculty members were used as controls. There were 52 controls from Istanbul and 34 from Leeds.

All HLA typings were performed in the Department of Immunohaematology of the University Hospital, Leiden, Holland. The typings were performed centrally in order to obviate any factors related to different antisera and methodology as causes for the differences in the previously mentioned studies.

Typing for the HLA A, B, and C specificities was performed with the standard NIH microcytotoxicity technique ${ }^{15}$ utilising a set of antisera for 13 A locus, $28 \mathrm{~B}$ locus, and $6 \mathrm{C}$ locus antigens. DR (D related) typing was performed with the 2-colour fluorescence test $^{16}$ using antisera for 10 DR specificities. Heparinised blood samples in siliconised bottles were 
transported by air from Istanbul and Leeds on the day of collection.

Differences in the antigen frequencies between patients and controls in the 2 populations were examined by means of the Woolf-Haldane tests. ${ }^{17} 18$

\section{Results}

The clinical findings for the patient groups are summarised in Tables $1,2 \mathrm{a}$, and $2 \mathrm{~b}$. There were no

Table 1 Incidence of various features in patients with Behçet's disease

\begin{tabular}{|c|c|c|c|c|}
\hline \multirow{4}{*}{$\begin{array}{l}\text { Aphthous stomatitis } \\
\text { Genital ulceration } \\
\text { Eye (anterior and } \\
\text { posterior segments) } \\
\text { Skin (erythema nodosum, } \\
\text { pyoderma, acne) } \\
\text { Arthritis }\end{array}$} & \multicolumn{2}{|c|}{ Istanbul } & \multicolumn{2}{|c|}{ Leeds } \\
\hline & $\begin{array}{l}22 \\
18\end{array}$ & $\begin{array}{r}(100 \%) \\
(82 \%)\end{array}$ & $\begin{array}{l}14 \\
14\end{array}$ & $\begin{array}{l}(100 \%) \\
(100 \%)\end{array}$ \\
\hline & 13 & $(59 \%)$ & 4 & $(28 \%)^{*}$ \\
\hline & $\begin{array}{l}21 \\
12\end{array}$ & $\begin{array}{l}(95 \%) \\
(54 \%)\end{array}$ & $\begin{array}{r}10 \\
8\end{array}$ & $\begin{array}{l}(71 \%) \\
(57 \%)\end{array}$ \\
\hline
\end{tabular}

*Including conjunctivitis in 1 British patient. major differences in the 2 patient populations except for a male predominance among the Turkish patients (19/22) as compared to the English patients (6/14). A breakdown of the age and sex information is set out in Table 3 .

Table 4 lists the distribution of HLA A, B, C, and DR specificities in the patient and control groups of both populations. The results of the WoolfHaldane analysis of B5 and its splits (Bw51 and Bw52) are set out in Table 5.

Although the distributions of the antigens B5, Bw51, and Bw52 in the Leeds population were not significantly different in the patient and control groups, they were not significantly heterogeneous when compared to the Istanbul population (all heterogeneity chi-squares less than $3 \cdot 84$ ). Consequently, the significance levels of $Y$ (relative risk) for B5 and Bw51 may be interpreted as reflecting true differences in the frequencies of those antigens in the patient and control groups of both populations.

Table 2a Features of patients with Behçet's disease in Istanbul

\begin{tabular}{|c|c|c|c|c|c|c|c|c|c|c|c|}
\hline \multirow{2}{*}{$\begin{array}{l}\text { Patient } \\
\text { no. }\end{array}$} & \multirow[t]{2}{*}{ Age } & \multirow[t]{2}{*}{ Sex } & \multirow{2}{*}{$\begin{array}{l}\text { Aphthous } \\
\text { stomatitis }\end{array}$} & \multirow{2}{*}{$\begin{array}{l}\text { Eye } \\
\text { lesions }\end{array}$} & \multirow{2}{*}{$\begin{array}{l}\text { Genital } \\
\text { lesions }\end{array}$} & \multirow{2}{*}{$\begin{array}{l}\text { Skin } \\
\text { lesions }\end{array}$} & \multirow[t]{2}{*}{ Arthritis } & \multirow[t]{2}{*}{ Special features } & \multicolumn{3}{|l|}{ HLA-typing } \\
\hline & & & & & & & & & $A B C$ & $D R$ & \\
\hline 1 & 30 & $\mathbf{M}$ & + & + & + & + & + & & $\begin{array}{l}\text { A2, A11, Bw51 } \\
\text { B 15B, w4, w6, Cw3 }\end{array}$ & w1 & LB47 \\
\hline 2 & 23 & $\mathbf{M}$ & + & + & + & + & + & & $\begin{array}{l}\text { A2, A9, Bw51, B40, } \\
\text { w4, w6, Cw3 }\end{array}$ & w6 & LB47 \\
\hline 3 & 21 & $\mathbf{M}$ & + & + & + & + & + & $\begin{array}{l}\text { Left ventricular } \\
\text { aneurysm }\end{array}$ & $\begin{array}{l}\text { A2, Aw24, Bw51, } \\
\text { B27, w4, Cw2 }\end{array}$ & w6 & w7 \\
\hline 4 & 36 & $\mathbf{M}$ & + & + & + & + & - & Thrombophlebitis & $\begin{array}{l}\text { A3, A11, Bw51, } \\
\text { w4, Cw2, Cw4 }\end{array}$ & LB47 & LB58 \\
\hline 5 & 37 & $\mathbf{M}$ & + & + & + & + & - & & $\begin{array}{l}\text { A2, A3, Bw51, } \\
\text { B18, w4, w6 }\end{array}$ & w2 & w5 \\
\hline 6 & 22 & $\mathbf{M}$ & + & + & - & + & - & & $\begin{array}{l}\text { A2, A3, Bw51, } \\
\text { B14, w4, w6, Cw6 }\end{array}$ & w5 & w6 \\
\hline 7 & 27 & $\mathbf{M}$ & + & + & + & + & - & $\begin{array}{l}\text { Superior vena } \\
\text { caval syndrome }\end{array}$ & $\begin{array}{l}\text { A1, Aw24, Bw51, } \\
\text { Bw35, w4, w6, Cw1 }\end{array}$ & w2 & w5 \\
\hline 8 & 47 & $\mathbf{F}$ & + & + & + & + & - & & $\begin{array}{l}\text { A1, A11, Bw51, } \\
\text { Bw35, w4, w6, Cw4 }\end{array}$ & nd & \\
\hline 9 & 45 & $\mathbf{M}$ & + & - & - & + & + & & $\begin{array}{l}\text { A26, Aw33, B18, B17, } \\
\text { w4, w6, Cw3 }\end{array}$ & w3 & w5 \\
\hline 10 & 36 & $\mathbf{M}$ & + & - & + & + & - & Thrombophlebitis & A2, Bw51, B17, W4 & w4 & w8 \\
\hline 11 & 29 & $\mathbf{M}$ & + & + & + & + & + & & $\begin{array}{l}\text { Aw24, Bw5 1, Bw35 } \\
\text { w4, w6, Cw4 }\end{array}$ & w5 & \\
\hline 12 & 33 & $\mathbf{F}$ & + & + & - & - & + & & $\begin{array}{l}\text { Aw24, B5, Bw35, } \\
\text { w4, w6, Cw4 }\end{array}$ & nd & \\
\hline 13 & 32 & $\mathbf{M}$ & + & - & + & + & + & & $\begin{array}{l}\text { A1, Aw24, Bw51, } \\
\text { B8, w4, w6 }\end{array}$ & w3 & w7 \\
\hline 14 & 41 & $\mathbf{M}$ & + & - & + & + & - & & $\begin{array}{l}\text { A3, A29, Bw51, } \\
\text { w4, Cw6 }\end{array}$ & w4 & w5 \\
\hline 15 & 37 & $\mathbf{M}$ & + & + & + & + & + & & $\begin{array}{l}\text { Aw30, Aw32, Bw52, } \\
\text { B7, w4, w6 }\end{array}$ & w4 & w6 \\
\hline 16 & 47 & $\mathbf{F}$ & + & - & + & + & + & & A29, Aw30, B14, w6 & w1 & \\
\hline 17 & 30 & $\mathbf{M}$ & + & + & + & + & + & Haemoptysis & $\begin{array}{l}\text { Aw24, Aw32, Bw51 } \\
\text { Bw44, w4 }\end{array}$ & w5 & LB58 \\
\hline 18 & 37 & $\mathbf{M}$ & + & - & + & + & - & & $\begin{array}{l}\text { A1, A2, B17 } \\
\text { w4, Cw6 }\end{array}$ & w5 & w7 \\
\hline 19 & 27 & $\mathbf{M}$ & + & + & + & + & - & & $\begin{array}{l}\text { A3, Aw24, Bw51, } \\
\text { Bw42, w4, w6 }\end{array}$ & w2 & w5 \\
\hline 20 & 37 & $\mathbf{M}$ & + & - & + & + & + & Sinus bradycardia & $\begin{array}{l}\text { A2, Bw5 1, Bw53, } \\
\text { w4, Cw4 }\end{array}$ & w6 & \\
\hline 21 & 23 & $\mathbf{M}$ & + & - & + & + & - & & $\begin{array}{l}\text { A28, Aw32, Bw51. } \\
\text { B18, w4, w6 }\end{array}$ & w5 & w6 \\
\hline 22 & 68 & $\mathbf{M}$ & + & - & - & + & + & & $\begin{array}{l}\text { A1, A3, Bw51, Bw44, } \\
\text { w4, Cw5 }\end{array}$ & nd & \\
\hline
\end{tabular}


Table 2b Features of patients with Behçet's disease in Leeds

\begin{tabular}{|c|c|c|c|c|c|c|c|c|c|c|}
\hline \multirow[t]{2}{*}{ Patient no. } & \multirow[t]{2}{*}{ Age } & \multirow[t]{2}{*}{$\operatorname{Sex}$} & \multirow{2}{*}{$\begin{array}{l}\text { Aphthous } \\
\text { stomatitis }\end{array}$} & \multirow{2}{*}{$\begin{array}{l}\text { Eye } \\
\text { lesions }\end{array}$} & \multirow{2}{*}{$\begin{array}{l}\text { Genital } \\
\text { lesions }\end{array}$} & \multirow{2}{*}{$\begin{array}{l}\text { Skin } \\
\text { lesions }\end{array}$} & \multirow[t]{2}{*}{ Arthritis } & \multirow[t]{2}{*}{ Special features } & \multicolumn{2}{|l|}{ HLA typing } \\
\hline & & & & & & & & & $A B C$ & $D R$ \\
\hline 1 & 33 & $\mathbf{M}$ & + & + & + & + & + & $\begin{array}{l}\text { Thrombophlebitis } \\
\text { Macular } \\
\text { haemorrhage }\end{array}$ & $\begin{array}{l}\text { A1, A11, Bw52, } \\
\text { B7, w4, w6 }\end{array}$ & w4 \\
\hline 2 & 29 & $\mathbf{M}$ & + & + & + & + & + & & $\begin{array}{l}\text { A1, A26, B8, B27, } \\
\text { w4, w6, Cw1 }\end{array}$ & w1 \\
\hline 3 & 41 & $\mathbf{F}$ & + & + & + & + & + & Arytenoid ulcer & $\begin{array}{l}\text { A2, Bw44, B15B, } 16 \\
\text { w4, w6, Cw3, Cw8 }\end{array}$ & $\begin{array}{l}\text { w5 } \\
\text { w7 }\end{array}$ \\
\hline 4 & 45 & $\mathbf{M}$ & + & - & + & + & - & Thrombophlebitis & $\begin{array}{l}\text { A2, Aw32, Bw51, : } \\
\text { B7, w4, w6, Cw2 }\end{array}$ & w4 \\
\hline 5 & 46 & $\mathbf{M}$ & + & - & + & + & + & $\begin{array}{l}\text { Thrombophlebitis } \\
\text { Tracheoesophageal } \\
\text { fistula }\end{array}$ & $\begin{array}{l}\text { A2, Bw51, 17, w4, } \\
\text { w6, Cw1, Cw5 }\end{array}$ & w6 \\
\hline 6 & 26 & $\mathbf{F}$ & + & - & + & + & - & & $\begin{array}{l}\text { A2, A11, Bw44, B15B, } 2 \\
\text { w4, w6, Cw1, Cw5 }\end{array}$ & w4 \\
\hline 7 & 38 & $\mathbf{F}$ & + & - & + & + & - & $\begin{array}{l}\text { Nonspecific } \\
\text { caecal ulceration }\end{array}$ & $\begin{array}{l}\text { A1, B7, B17, } \\
\text { w4, w6, Cw6 }\end{array}$ & w4 \\
\hline 8 & 43 & $\mathbf{F}$ & + & - & + & + & - & & $\begin{array}{l}\text { Aw24, A28, Bw44, } \\
\text { w4, Cw5 }\end{array}$ & nd \\
\hline 9 & 14 & $\mathbf{M}$ & + & - & + & + & + & & $\begin{array}{l}\text { A2, A11, Bw22, B40, } \\
\text { w6, Cw3 }\end{array}$ & w2 \\
\hline 10 & 51 & $\mathbf{F}$ & + & $+(c)$ & + & - & - & & $\begin{array}{l}\text { A2, A11, Bw35, B27, } \\
\text { w4, w6, Cw2, Cw4 }\end{array}$ & $\begin{array}{l}\text { w4 } \\
\text { w6 }\end{array}$ \\
\hline 11 & 35 & $\mathbf{F}$ & + & - & + & - & + & & $\begin{array}{l}\text { A1, Aw24, B8, B27, } \\
\text { w4, w6, Cw1 }\end{array}$ & $\begin{array}{l}\text { w1 } \\
\text { w4 }\end{array}$ \\
\hline 12 & 66 & $\mathbf{F}$ & + & - & + & - & + & & $\begin{array}{l}\text { A2, Aw32, B18, B40 } \\
\text { w6, Cw3 }\end{array}$ & nd \\
\hline 13 & 38 & $\mathbf{F}$ & + & - & + & - & + & Thrombophlebitis & $\begin{array}{l}\text { A2, B27, B40 } \\
\text { w4, w6, Cw3 }\end{array}$ & w4 \\
\hline 14 & 60 & $\mathbf{M}$ & + & + & + & + & - & $\begin{array}{l}\text { Paget's disease of } \\
\text { skull also }\end{array}$ & $\begin{array}{l}\text { A1, 2, Bw51 } \\
37, \text { w4, w4, Cw6 }\end{array}$ & $\begin{array}{l}\text { w3 } \\
\text { w4 }\end{array}$ \\
\hline
\end{tabular}

(c) = Conjunctivitis only.

Table 3 Distributions of age and sex in the Istanbul and Leeds Behçet patient populations

\begin{tabular}{llrlll}
\hline \multirow{5}{*}{ No. } & \multicolumn{2}{l}{ Age } & \\
\cline { 3 - 6 } & & & Range & Mean & Median \\
\hline Istanbul & Males & 19 & $21-68$ & $33 \cdot 6$ & 30 \\
\multirow{4}{*}{ Leeds } & Females & 3 & $33-47$ & $42 \cdot 3$ & 47 \\
& Males & 6 & $14-60$ & $36 \cdot 2$ & 33 \\
& Females & 8 & $26-66$ & $42 \cdot 3$ & 41 \\
\hline
\end{tabular}

Table 4 Distribution of $H L A A, B, C$, and $D R$ specificities in patient and control groups

\begin{tabular}{|c|c|c|c|c|}
\hline \multirow[t]{2}{*}{$H L A$} & \multicolumn{2}{|l|}{ Istanbul } & \multicolumn{2}{|l|}{ Leeds } \\
\hline & $\begin{array}{l}\text { Patients } \\
N=22\end{array}$ & $\begin{array}{l}\text { Controls } \\
N=52\end{array}$ & $\begin{array}{l}\text { Patients } \\
N=14\end{array}$ & $\begin{array}{l}\text { Controls } \\
N=34\end{array}$ \\
\hline $\begin{array}{l}\text { A1 } \\
\text { A2 } \\
\text { A3 } \\
\text { A9 } \\
\text { A10 } \\
\text { A11 } \\
\text { A28 } \\
\text { A29 } \\
\text { Aw30 } \\
\text { Aw31 } \\
\text { Aw32 } \\
\text { Aw33 } \\
\text { Aw34 }\end{array}$ & $\begin{array}{l}5^{*} \\
8 \\
5 \\
7 \\
1 \\
3 \\
1 \\
2 \\
2 \\
0 \\
3 \\
2 \\
0\end{array}$ & $\begin{array}{r}10 \\
27 \\
13 \\
16 \\
7 \\
5 \\
5 \\
0 \\
3 \\
1 \\
4 \\
4 \\
0\end{array}$ & $\begin{array}{l}5 \\
9 \\
0 \\
2 \\
1 \\
3 \\
1 \\
0 \\
0 \\
0 \\
2 \\
0 \\
0\end{array}$ & $\begin{array}{r}13 \\
19 \\
9 \\
6 \\
1 \\
6 \\
1 \\
3 \\
1 \\
4 \\
0 \\
0 \\
2\end{array}$ \\
\hline
\end{tabular}

* Number of positive individuals.

Table $4 b$

\begin{tabular}{|c|c|c|c|c|}
\hline \multirow[t]{2}{*}{$H L A$} & \multicolumn{2}{|l|}{ Istanbul } & \multicolumn{2}{|l|}{ Leeds } \\
\hline & $\begin{array}{l}\text { Patients } \\
N=22\end{array}$ & $\begin{array}{l}\text { Controls } \\
N=52\end{array}$ & $\begin{array}{l}\text { Patients } \\
N=14\end{array}$ & $\begin{array}{l}\text { Controls } \\
N=34\end{array}$ \\
\hline B5 & 19 & 22 & 4 & 6 \\
\hline Bw51 & 18 & 12 & 3 & 4 \\
\hline Bw52 & 1 & 9 & 1 & 2 \\
\hline B7 & i & 4 & 3 & 12 \\
\hline B8 & 1 & 5 & 2 & 9 \\
\hline B13 & 0 & 3 & 0 & 2 \\
\hline B14 & 2 & 1 & 0 & 0 \\
\hline B15 A & 0 & 1 & 0 & 1 \\
\hline B15 B & 1 & 0 & 2 & 1 \\
\hline B17 & 3 & 6 & 2 & 1 \\
\hline B18 & 3 & 7 & 1 & 1 \\
\hline Bw22 & 0 & 2 & 1 & 5 \\
\hline B27 & 1 & 3 & 4 & 1 \\
\hline Bw35 & 4 & 11 & 1 & 5 \\
\hline B37 & 0 & 1 & i & 1 \\
\hline Bw38 & 0 & 6 & 0 & 0 \\
\hline Bw39 & 0 & 2 & 0 & 1 \\
\hline B40 & 1 & 4 & 3 & 4 \\
\hline Bw41 & 0 & 2 & 0 & 1 \\
\hline Bw42 & 1 & 0 & 0 & 0 \\
\hline Bw44 & 2 & 6 & 3 & 9 \\
\hline Bw45 & 0 & o & 0 & 0 \\
\hline Bw47 & 0 & 0 & O & 0 \\
\hline Bw48 & 0 & 1 & 0 & 0 \\
\hline Bw49 & o & 5 & 0 & 1 \\
\hline Bw50 & 0 & 4 & 0 & 1 \\
\hline Bw53 & o & 2 & 0 & 0 \\
\hline
\end{tabular}


Table 4c

\begin{tabular}{|c|c|c|c|c|}
\hline \multirow[t]{2}{*}{$H L A$} & \multicolumn{2}{|l|}{ Istanbul } & \multicolumn{2}{|l|}{ Leeds } \\
\hline & $\begin{array}{l}\text { Patients } \\
N=22\end{array}$ & $\begin{array}{l}\text { Controls } \\
N=52\end{array}$ & $\begin{array}{l}\text { Patients } \\
N=14\end{array}$ & $\begin{array}{l}\text { Controls } \\
N=34\end{array}$ \\
\hline Cw1 & 1 & 2 & 3 & 1 \\
\hline Cw2 & 2 & 4 & 2 & 1 \\
\hline Cw3 & 3 & 6 & 4 & 9 \\
\hline Cw4 & 5 & 14 & 1 & 3 \\
\hline Cw5 & 1 & 2 & 3 & 1 \\
\hline \multirow[t]{2}{*}{ Cw6 } & 3 & 9 & 2 & 5 \\
\hline & $N=19$ & $N=49$ & $N=12$ & $N=28$ \\
\hline DRw1 & 2 & 11 & 2 & 6 \\
\hline DRw2 & 3 & 13 & 1 & 8 \\
\hline DRw3 & 2 & 10 & 1 & 5 \\
\hline DRw4 & 3 & 11 & 8 & 12 \\
\hline DRw5 & 10 & 18 & 1 & 2 \\
\hline DRw6 & 6 & 12 & 2 & 10 \\
\hline DRw7 & 3 & 11 & 1 & 5 \\
\hline LB8 & 1 & 1 & 0 & 0 \\
\hline LB47 & 3 & 6 & 0 & 0 \\
\hline LB58 & 2 & 3 & 0 & 0 \\
\hline
\end{tabular}

It is interesting to note that all of the English Behçet's patients with B5 are male.

The number of observations for the DR antigens were small. They have been analysed and listed in Table 5 because of the reported linkage disequilibrium between B5 and DRw2 and DRw4 in North American caucasoids. ${ }^{19}$ There is no difference in the incidence of DRw2 and DRw4 in Turkish and British patients when compared with their controls.

Since the numbers of observations were rather small for the remaining HLA and A, B, C, and DR specificities, the frequency differences were not analysed, in order to avoid the effect of sampling errors.

The data in this study were insufficient to confirm our previously reported finding of a weak association between B27 and English patients with Behçet's disease.

\section{Discussion}

The results of this study demonstrate significant conformity in the different incidence of HLA B5, specifically its Bw51 split, in patients with Behçet's disease in 2 Caucasoid populations. The modest increase in the frequency of HLA B27 in British patients is suggested by the data but was not analysed because of the small sample sizes.

The striking increase in the frequency of HLA B5 among the Turkish patients is confined to Bw51, the split of B5 found commonly among Caucasians. ${ }^{12}$ It is interesting that the relative risk statistic for B5 in Caucasians was reported as 7.43 by the HLA and Disease Registry in $1977 .{ }^{20}$ The value is $7 \cdot 55$ for the same antigen in the Istanbul population. However, it is almost twice as large for Bw51 in the same population (see Table 4). These observations suggest that other investigators should type their Behçet patients for the B5 splits. This would be especially important for the Japanese patients, since Bw51 and Bw52 seem to be common alleles in the Japanese population. ${ }^{12}$

An inspection of the report of the HLA and Disease Registry reveals very few disease associations with antigenic splits. In view of the findings in this report it is perhaps pertinent to suggest that a reappraisal of HLA and disease associations with special attention to antigenic splits may reveal new associations or strengthen presently recognised associations.

We acknowledge with thanks the help of Professor J. J. van Rood and Professor V. Wright, Mrs K. Burton, Dr C. Eastmond, and Mrs J. Packter. We thank the patients who participated and the Royal Dutch Airlines, which transported specimens from Turkey.

This work was in part supported by the National Institutes of Health (contract no. NO1-A1-4-2508), the Dutch Organisation for Health Research (TNO), the Dutch Foundation for Medical Research (FUNGO), which is subsidised by the Dutch Foundation for the Advancement of Pure Research (ZWO), the J. A. Cohen Institute for Radiopathology and Radiation Protection (IRS), and the Turkish Scientific and Technical Research Council, TUBITAK (TAG-386).

\section{References}

1 Ohno S, Aoki K, Sugiura S, Nakayama E, Itakura K, Aizawa M. HL-A5 and Behçet's disease. Lancet 1973; 2: $1383-4$.

Table 5 Woolf-Haldane analysis of HLA B5 and its splits [Bw51 and Bw52] in 2 patient populations and their controls

\begin{tabular}{|c|c|c|c|c|c|c|c|c|c|c|c|c|c|}
\hline & \multicolumn{5}{|l|}{ Istanbul } & \multicolumn{5}{|l|}{ Leeds } & \multirow{2}{*}{\multicolumn{2}{|c|}{ Chi squares }} & \multirow[b]{2}{*}{ Total } \\
\hline & $\begin{array}{l}\text { Patients } \\
\text { Pos. }\end{array}$ & Neg. & $\begin{array}{l}\text { Controls } \\
\text { Pos. }\end{array}$ & Neg. & $Y^{1}$ & $\begin{array}{l}\text { Patients } \\
\text { Pos. }\end{array}$ & Neg. & $\begin{array}{l}\text { Controls } \\
\text { Pos. }\end{array}$ & Neg. & $Y$ & & & \\
\hline $\begin{array}{l}\text { B5 } \\
\text { Bw51 } \\
\text { Bw52 } \\
\text { DRw2 } \\
\text { DRw4 }\end{array}$ & $\begin{array}{r}19 \\
18 \\
1 \\
3 \\
3\end{array}$ & $\begin{array}{r}3 \\
4 \\
21 \\
16 \\
16\end{array}$ & $\begin{array}{r}22 \\
12 \\
9 \\
13 \\
11\end{array}$ & $\begin{array}{l}30 \\
40 \\
43 \\
36 \\
38\end{array}$ & $\begin{array}{r}7 \cdot 552 \\
13 \cdot 320 \\
0 \cdot 319 \\
0 \cdot 574 \\
0 \cdot 710\end{array}$ & $\begin{array}{l}4 \\
3 \\
1 \\
1 \\
8\end{array}$ & $\begin{array}{r}10 \\
11 \\
13 \\
11 \\
4\end{array}$ & $\begin{array}{r}6 \\
4 \\
2 \\
8 \\
12\end{array}$ & $\begin{array}{l}28 \\
30 \\
32 \\
20 \\
16\end{array}$ & $\begin{array}{l}1 \cdot 879 \\
2 \cdot 063 \\
1 \cdot 444 \\
0.315 \\
2 \cdot 493\end{array}$ & $\begin{array}{c}9.4373 \\
16 \cdot 085^{4} \\
0.673 \\
2 \cdot 248 \\
0 \cdot 324\end{array}$ & $\begin{array}{l}2 \cdot 293 \\
3 \cdot 783 \\
1 \cdot 420 \\
0 \cdot 314 \\
1 \cdot 824\end{array}$ & $\begin{array}{r}11 \cdot 730 \\
19 \cdot 869 \\
2 \cdot 093 \\
2 \cdot 561 \\
2 \cdot 148\end{array}$ \\
\hline
\end{tabular}

${ }^{1} \mathbf{Y}=$ the relative risk statistic. ${ }^{2} \mathrm{Het} .=$ Heterogeneity. $\quad{ }^{3} \mathbf{P}=0.009 . \quad{ }^{4} \mathbf{P}=0.0003$. 
2 Ersoy F, Berkel A I, Firat T, Kazokoglu H. HLA antigen associated with Behçet's disease. HLA and Disease. Paris: Editions INSERM, 1976.

3 Yazici H, Akokan G, Yalcin B, Muftuoglu A. The high prevalence of HLA-B5 in Behçet's disease. Clin Exp Immunol 1977; 30: 259-61.

4 Chajek T, Brautbar C, Cohen T, Lamm L U. A study of genetic factors in patients with Behçet's disease in Israel. Aba No. 907, 1977.

5 Godeau P, Terre D, Campinchi R, et al. HLA-B5 and Behçet's disease. HLA and Disease, 101. Paris: Editions INSERM, 1976.

- Takano M, Miyajima Y, Kiuchi M. et al. Behçet's Disease and the HLA system. Tissue Antigens 1976; 8: 95-9.

7 Hamza M. International Symposium on Behçet's Disease. Istanbul, 1977.

8 Chamberlain M A. (1975). Behçet's disease. Ann Rheum Dis 1975; 34: Suppl 1, 53-4.

- Jung R T, Chalmers T M, Joysey V C. HLA in Behçet's disease. Lancet 1976; 1 : 694.

10 O'Duffy J D, Taswell H F, Elvebach L R. HLA antigens in Behçet's disease. $J$ Rheumatol 1976; 3: 1-8.

11 Chamberlain M A. Behçet's syndrome in 32 patients in Yorkshire. Ann Rheum Dis 1977; 36: 491-9.
12 Dick H. HLA-A, B, and C serology and antigen report. In Histocompatability Testing 1977. Joint Report, 157. Copenhagen: Munksgaard, 1978.

13 Mason R M, Barnes C G. Behcet's syndrome with arthritis. Ann Rheum Dis 1969; 28: 95-103.

14 O'Duffy J D. Suggested criteria for diagnosis of Behçet's disease. Book of Abstracts, Toronto, Canada: VI Pan American Congress of Rheumatic Disease, 18, 1974.

15 Manual of Tissue Typing Techniques (1976-77). Department of Health, Education and Welfare. Publications No. (NIH) 77-545.

16 Rood J J van, Loouwen A van, Ploem J S. Simultaneous detection of two cell populations by two-colour fluorescence and application to the recognition of B-cell determinants. Nature 1976; 262: 795-7.

17 Woolf B. (1955). On estimating the relation between blood groups and disease. Ann Hum Genet 19, 251-253.

18 Haldane J B S. The estimation and significance of the logarithm of a ratio of frequencies. Ann Hum Genet 1955; 20 : 309-11.

19 Pickbourne P, Piazza A, Bodmer F. Population Analysis. In Histocompatibility Testing 1977. Joint Report, 259 Copenhagen: Munksgaard, 1978.

20 Svejgaard A, Ryder L P. HLA and disease, In: Ferrara G B, ed. HLA system-new aspects. Amsterdam: North Holland, 1977; 143-51. 